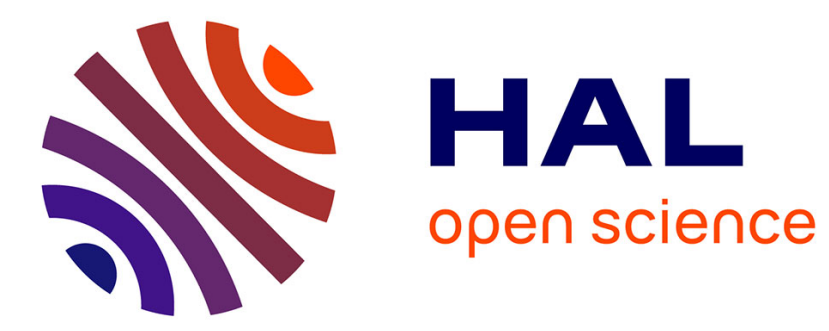

\title{
Boson representations for systems of fermions
}

Jean-Paul Blaizot, Henri Orland

\section{To cite this version:}

Jean-Paul Blaizot, Henri Orland. Boson representations for systems of fermions. Journal de Physique Lettres, 1980, 41 (22), pp.523-525. 10.1051/jphyslet:019800041022052300 . jpa-00231837

\section{HAL Id: jpa-00231837 https://hal.science/jpa-00231837}

Submitted on 1 Jan 1980

HAL is a multi-disciplinary open access archive for the deposit and dissemination of scientific research documents, whether they are published or not. The documents may come from teaching and research institutions in France or abroad, or from public or private research centers.
L'archive ouverte pluridisciplinaire HAL, est destinée au dépôt et à la diffusion de documents scientifiques de niveau recherche, publiés ou non, émanant des établissements d'enseignement et de recherche français ou étrangers, des laboratoires publics ou privés. 


\title{
LE JOURNAL DE PHYSIQUE-LETTRES
}

J. Physique - LETTRES 41 (1980) L-523 - L-525

15 NOVEMBRE 1980, PAGE L-523

Classification

Physics Abstracts

$03.65-21.60 \mathrm{~J}$

\section{Boson representations for systems of fermions}

\author{
J. P. Blaizot and H. Orland \\ Service de Physique Théorique, CEN Saclay, BP No 2, 91190 Gif sur Yvette, France \\ (Reçu le 11 juillet 1980, accepté le 26 septembre 1980)
}

\begin{abstract}
Résumé. - Nous présentons une description des systèmes de fermions en termes de degrés de liberté bosoniques qui permettent de quantifier exactement les équations de champ moyen. Nous montrons que cette représentation bosonique généralise celles déjà utilisées.
\end{abstract} Abstract. - We present a new boson representation for systems of fermions. This representation allows a direct
quantization of the mean field equations. The relation to existing boson representations is discussed.

The time dependent Hartree-Fock approximation leads to equations of motion which can be interpreted as classical field equations [2]. In order to recover the original many-body problem, a quantization of these classical fields has to be performed. This introduces boson degrees of freedom, and depending upon the choice of the classical fields, the quantization leads to different boson representation of the original fermion Hamiltonian [3]. In this paper we present a new representation, which appears as a generalization of the work of reference [1]. We show how this representation is suited for a direct quantization of the time dependent Hartree equations. The quantization of the time dependent Hartree-Fock equations requires a specific treatment and will be presented in a separate paper [4].

We consider a system of fermions with the Hamiltonian :

$$
\begin{aligned}
H=\sum_{\alpha \beta} H_{\alpha \beta}^{0} a_{\alpha}^{+} a_{\beta}+\frac{1}{2} \sum_{\alpha \beta \gamma \delta}(\alpha \beta|V| \gamma \delta) \times & \\
& \times a_{\alpha}^{+} a_{\beta}^{+} a_{\delta} a_{\gamma}
\end{aligned}
$$

where $(\alpha \beta|V| \gamma \delta)$ is a non-antisymmetrized matrix element of the two-body interaction $V$. The creation and annihilation operators satisfy fermion anticommutation rules :

$$
\left[a_{\alpha}, a_{\beta}^{+}\right]_{+}=\delta_{\alpha \beta} .
$$

The expression (1) is the standard second quantized form of the Hamiltonian in the fermion Fock space $\mathcal{F}$. This Fock space $\mathcal{F}$ is built from the Hilbert space $\mathfrak{H}_{1}$ of single particle states $|\alpha\rangle,|\beta\rangle \ldots$ From the same single particle Hilbert space $\mathfrak{H}_{1}$, one can also cons- truct a boson Fock space $\mathfrak{B}$. We denote by $C_{\alpha}^{+}, C_{\beta}$ the boson creation and annihilation operators. They satisfy boson commutation rules :

$$
\left[C_{\alpha}, C_{\beta}^{+}\right]=\delta_{\alpha \beta} .
$$

Let $|0\rangle$ and $\mid 0$ ) be the vacuum states in $\mathcal{F}$ and $\mathcal{B}$ respectively. It is clear that a one-to-one mapping can be realized between the states of $\mathcal{F}$ and $\mathscr{B}$ containing only one particle : the same single particle wave function $\varphi_{\alpha}(x)$ is associated to the states $a_{\alpha}^{+}|0\rangle$ and $\left.C_{\alpha}^{+} \mid 0\right)$. Thus boson states containing only one particle may be chosen to represent one particle fermion states. To construct a boson image of an $\mathrm{N}$-fermions state, we consider a large space $\mathrm{G}$, product of $N$ boson Fock space $\mathcal{B}_{i}$ associated with each of the particle $i$ :

$$
\mathrm{G}=\mathfrak{B}_{1} \otimes \mathfrak{B}_{2} \otimes \cdots \otimes \mathfrak{B}_{N} .
$$

We call $C_{i}^{+}(\alpha), C_{i}(\alpha)$ the creation and annihilation operators acting in the space $\mathcal{B}_{i}$. These operators satisfy the following commutation rules :

$$
\begin{aligned}
{\left[C_{i}(\alpha), C_{j}^{+}(\beta)\right] } & =\delta_{i j} \delta_{\alpha \beta} \\
{\left[C_{i}(\alpha), C_{j}(\beta)\right] } & =\left[C_{i}^{+}(\alpha), C_{j}^{+}(\beta)\right]=0 .
\end{aligned}
$$

The following states :

$$
\sum_{P}(-)^{P} C_{1}^{+}\left(\alpha_{P_{1}}\right) \ldots C_{N}^{+}\left(\alpha_{P_{N}}\right) \mid 0
$$

where $\sum_{P}$ is a sum over all possible permutations of the indices $\alpha_{1} \ldots \alpha_{N}$, are in one-to-one correspondence with the $N$-fermions states in $\mathcal{F}$. In this way, one can 
map the fermion Fock space into a subspace of $\mathrm{G}$, called the physical subspace. The states which belong to the physical subspace are characterized by two properties. They are antisymmetric and there is one and only one particle per subspace $\mathfrak{B}_{i}$.

The operators in the fermion Fock space $\mathcal{F}$ are extended into operators acting in the large space $G$. The extension is defined in such a way that the matrix element of a given operator in the physical subspace of $G$ are identical with the matrix elements of the corresponding operator in the fermion Fock space. We consider explicitly one- and two-body operators. It is easily verified that :

$$
\tilde{W}=\sum_{\alpha \beta} W_{\alpha \beta} \sum_{i=1}^{N} C_{i}^{+}(\alpha) C_{i}(\beta)
$$

acts in the same way on the physical states as the operator $W=\sum_{\alpha \beta} W_{\alpha \beta} a_{\alpha}^{+} a_{\beta}$ does in the fermion

Fock space. In the same way, the extension of a twobody operator is :

$\tilde{V}=\frac{1}{2} \sum_{i j} \sum_{\alpha \beta \gamma \delta}(\alpha \beta|V| \gamma \delta) C_{i}^{+}(\alpha) C_{j}^{+}(\beta) C_{j}(\delta) C_{i}(\gamma)$.

The operator which counts the number of particles in the space $\mathcal{B}_{i}$ is :

$$
N_{i}=\sum_{\alpha} C_{i}^{+}(\alpha) C_{i}(\alpha)
$$

The physical states are eigenstates of the operator $N_{i}$ with eigenvalue 1 . Actually, they obey a more general equation, which proves to be useful in some applications :

$$
\sum_{\alpha} C_{i}^{+}(\alpha) C_{j}(\alpha)|\phi\rangle=\delta_{i j}|\phi\rangle
$$

It is important to notice that the operator which realizes the constraint (10) commutes with the oneand two-body operators $\tilde{W}$ and $\tilde{V}$ given by equations (7) and (8) respectively. Furthermore these operators are symmetric in the coordinates of the particles and therefore commute with the operator of antisymmetrization. It follows that the operators $\tilde{V}$ and $\widetilde{W}$ have no matrix elements between physical and unphysical states. Thus the solution to the Schrödinger equation for the fermions, can be looked for either directly in the fermion Fock space, or by projecting on the physical subspace a solution obtained in the large space $\mathrm{G}$. The interest of boson representations lies of course in that they may offer new approximation schemes.

As an example of application of the preceding formalism, let us consider the equation of motion for the operator $C_{k}(v)$ in the large space $\mathrm{G}$ :

$$
i \frac{\mathrm{d}}{\mathrm{d} t} C_{k}(v)=\sum_{\beta} \hat{h}_{v \beta} C_{k}(\beta)
$$

with

$$
\hat{h}_{\nu \beta}=W_{\nu \beta}+\sum_{i} \sum_{\alpha \gamma}(\alpha \nu|V| \gamma \beta) C_{i}^{+}(\alpha) C_{i}(\gamma) .
$$

An approximation to these equations consists in replacing the boson operators by $C$-numbers. This is the so-called classical limit. The meaning of this classical limit is most clearly seen if we go to a representation in terms of field operators. Then equation (11) reads :

$$
i \frac{\mathrm{d}}{\mathrm{d} t} \psi_{k}(x)=\int \mathrm{d} x^{\prime}\left\langle x|\hat{h}| x^{\prime}\right\rangle \psi_{k}\left(x^{\prime}\right) .
$$

Replacement of the field operators $\psi_{k}(x)$ by $C$-numbers, i.e. wave functions $\varphi_{k}(x)$, yields the familiar time dependent Hartree equations. The wave function $\varphi_{k}(x)$ can be considered as the eigenvalue of the field operator $\psi_{k}(x)$, the eigenvector being the coherent state :

$$
\left.\left.\mid \varphi_{k}\right)=\mathrm{e}^{\int \varphi_{k}(x) \psi_{k}^{+}(x)} \mid 0\right) .
$$

Quantization of the time dependent Hartree equations means simply reversing the procedure, i.e. replacing the single particle wave functions by boson field operators and taking care of the constraints which project on the physical subspace. The quantization of the time dependent Hartree equations along these lines has been widely discussed in reference [1] which presents great analogy with the present work. The coherent states (14) are useful for constructing path integrals for the many-body problem. This will be discussed in an other paper (Ref. [3]). We also discuss separately the problem associated with the quantization of the time dependent Hartree-Fock equations.

We conclude this note by a simple, though non trivial, example which illustrates how bosons coherent states may be used in a problem of fermions.

A somewhat analogous phenomenon has been recently reported in reference [6]. We shall even restrict ourselves to the case of a single particle and calculate the partition function :

$$
Z=\operatorname{Tr} \mathrm{e}^{-\beta H}=\sum_{n} \mathrm{e}^{-\beta \varepsilon_{n}}
$$

where on the right hand side the trace has been calculated using the eigenstates of the Hamiltonian $H$. The trace can be calculated differently, and for example using the physical states of the large space $G$ :

$$
Z=\operatorname{Tr}_{\mathrm{G}} P \mathrm{e}^{-\beta H}
$$

where $P$ is the projector on the physical subspace. In the present case :

$$
P=\int_{-\pi}^{\pi} \frac{\mathrm{d} \theta}{2 \pi} \mathrm{e}^{i(N-1) \theta}
$$

where $N$ is the number of particles operator. To eva- 
luate the trace it is convenient to use the coherent states :

$$
\left.|\zeta\rangle=\mathrm{e}_{\alpha}^{\Sigma \zeta \alpha} C_{\alpha}^{+} \mid 0\right) .
$$

These states form an overcomplete basis of G. The decomposition of unity reads :

$$
\int \prod_{\alpha} \frac{\mathrm{d} \zeta_{\alpha}^{*} \mathrm{~d} \zeta_{\alpha}}{2 \pi i} \mathrm{e}^{-\Sigma_{\alpha} \zeta_{\alpha}^{*} \zeta_{\alpha}}|\zeta\rangle\langle\zeta|=1
$$

This relation can be used to construct a functional representation for $Z$ :

$$
\begin{aligned}
Z=\int_{-\pi}^{\pi} \frac{\mathrm{d} \theta}{2 \pi} \mathrm{e}^{-i \theta} \int_{Z_{\alpha}(\beta)=Z_{\alpha}(0)} \prod_{\alpha} \mathcal{D}\left(Z_{\alpha}^{*}, Z_{\alpha}\right) \times \\
\times \exp \left[-\int_{0}^{\beta} Z_{\alpha}^{*}\left(\partial_{t}+\varepsilon_{\alpha}-i \frac{\theta}{\beta}\right) Z_{\alpha}\right] .
\end{aligned}
$$

The integration over the variables $Z_{\alpha}^{*}, Z_{\alpha}$ can be performed explicitly and yields :

$Z=\int_{-\pi}^{\pi} \frac{\mathrm{d} \theta}{2 \pi} \mathrm{e}^{-i \theta} \prod_{\alpha}\left[\operatorname{Det}\left(\partial_{t}+\varepsilon_{\alpha}-i \frac{\theta}{\beta}\right)\right]^{-1}$.

The determinant is then calculated by diagonalizing the operator $\partial_{t}+\varepsilon_{\alpha}-i \frac{\theta}{\beta}$. One finally obtains :

$$
Z=\int_{-\pi}^{\pi} \frac{\mathrm{d} \theta}{2 \pi} \mathrm{e}^{-i \theta} \prod_{\alpha}\left(\frac{1}{1-\mathrm{e}^{-\beta \varepsilon_{\alpha}} \mathrm{e}^{i \theta}}\right) .
$$

One recognizes in the integrand of (22) the partition function for a non interacting system of bosons with a chemical potential equal to $i \frac{\theta}{\beta}$. The integration over $\theta$ projects out the components with only one boson present in each state $\alpha$ and retrieves the formula (15).

Functional integral representations for fermions are usually expressed in terms of Grassman numbers [5]. In this case, the partition function (15) can be written :

$$
\begin{aligned}
Z=\int_{-\pi}^{+\pi} \frac{\mathrm{d} \theta}{2 \pi} \mathrm{e}^{-i \theta} \int_{\xi_{\alpha}(\beta)=-\xi_{\alpha}(0)} \prod_{\alpha} D\left(\xi_{\alpha}^{*}, \xi_{\alpha}\right) \times \\
\quad \times \exp \left[-\int_{0}^{\beta} \mathrm{d} t_{\alpha}^{*}\left(\partial_{t}+\varepsilon_{\alpha}-i \frac{\theta}{\beta}\right) \xi_{\alpha}\right]
\end{aligned}
$$

The integration over the Grassman variables can be performed, and yields :

$$
Z=\int_{-\pi}^{+\pi} \frac{\mathrm{d} \theta}{2 \pi} \mathrm{e}^{-i \theta} \prod_{\alpha}\left(1+\mathrm{e}^{-\beta \varepsilon_{\alpha}} \mathrm{e}^{i \theta}\right)
$$

which is equal to (22), after integration over $\theta$.

This example illustrates on a very simple case how one can transform calculations in a fermion Hilbert space into calculation in boson Fock space. This sometimes proves to be helpful in some complicated problems. It is extensively used in the construction of path integrals for the many-body problem. There are two reasons which make the use of boson states useful in the context of path integral. The first one is that the projector onto the physical subspace commutes with the Hamiltonian and therefore needs not be inserted at each time step. The second reason is that boson coherent states are eigenstates of the field operators, which facilitates the calculation of the matrix elements.

\section{References}

[1] Blaizot, J. P. and MarshaleK, E. R., Phys. Lett. 79B (1978) 1 ; Nucl. Phys. A 309 (1978) and A 309 (1978) 453.

[2] Kerman, A. K. and Koonin, S., Ann. Phys. 100 (1976) 332.

[3] Rowe, D. J. and Bassermann, R., Can. J. Phys. 54 (1976) 1942; Marumori, T., Yamamura, M. and Tokunaga, A., Prog. Theor. Phys. 31 (1964) 1009.

SCHWINGER, J., in Quantum Theory of Angular Momentum, eds. L. Biedenharn and H. Van Dam (Academic Press, New York) 1965, p. 229.
Holstein, T. and Primakoff. H., Phys. Rev. 58 (1950) 1098.

[4] Blaizot, J. P. and Orland, H., submitted to Phlis. Lett. and in preparation. See also J. Physique Lett. 41 (1980) L-53.

[5] Fadeev, L., Methods in Quantum Field Theory, Les Houches (1975), R. Balian and J. Zinn-Justin eds. (North Holland Publishing Company)

Ohnuki, Y. and Kashiwa, T., Prog. Theor. Phys. 60 (1978) 548.

[6] Haka, H. and Kugo, T., Phys. Rev. D 21 (1980) 3333. 